\title{
Uso de las TIC en la Práctica Pedagógica de los Docentes Rurales en Colombia
}

\author{
María Patricia Gómez Becerraํ, Gloria Elizabeth Bernal De Felipe ${ }^{2}$, \\ Carolina Medrano León ${ }^{3}$
}

Recepción: 14-10-2014

Aceptación: 10-01-2015

\section{Resumen}

El artículo presenta los resultados de un estudio descriptivo llevado a cabo en veintiún instituciones educativas rurales del departamento de Cundinamarca en Colombia. El propósito fue indagar sobre los usos de las TIC en las prácticas pedagógicas de un grupo de profesores que atienden desde el nivel preescolar hasta básica primaria. La investigación utilizó un enfoque mixto con técnicas cualitativas y cuantitativas. El análisis de la información muestra que los maestros vinculados a las sedes rurales estudiadas, están en un nivel básico de apropiación con respecto al uso de las TIC. Sin embargo, es importante tener en cuenta que hay un progreso en la transición de algunos de estos maestros al nivel de apropiación intermedio, especialmente en el uso de las TIC para proponer y ejecutar proyectos de aula.

Palabras clave: Formación de docentes, TIC, uso de las TIC, prácticas pedagógicas y educación rural

\begin{abstract}
The article presents the results of a descriptive study carried out in twenty one rural educational institutions of the department of Cundinamarca, Colombia. The purpose was to explore the uses of the ICT in the teaching practices of a group of teachers that attend from pre-school students to basic primary level. The investigation used a mix-approach with quantitative and qualitative techniques. The analysis of the information shows that the teachers involved in those rural educational institutions studied are in a basic level of appropriation regarding the use of the ICT. However, it is important to have in mind that there is no progress in the transition of some of those teachers to the intermediate appropriation level especially in the use of the ICT to propose and carry out projects for classrooms.
\end{abstract}

Key words: forming teachers, ICT, use of the ICT, teaching practices and rural education

1. Máster en Educación, Profesora Universidad de La Sabana, Facultad de Educación, maria.gomez1@ unisabana.edu.co

2. Máster en Pedagogía, Auditora pedagógica, Fundación para el Fomento de la Lectura- FUNDALECTURA, gloria.bernal1@unisabana.edu.co

3. Máster en Educación, Coordinadora pedagógica de proyecto, Unión Temporal Universidad de la Sabana- FES, carolina.medrano@unisabana.edu.co 


\section{Introducción}

El fenómeno de las TIC ha traído consigo un gran impacto a nivel mundial, tanto así que la mayoría de los países han tenido que reformar sus sistemas educativos para poder adaptarlos a las exigencias de la sociedad en este campo.

En Colombia, el ingreso de la informática se dio de una forma rápida e inmediata. Hoy son cada vez más los colombianos que acceden al mundo de la tecnología como una posibilidad de progresar, aumentar sus oportunidades de negocios, de trabajo, de estudio y de cultura para mejorar sus ingresos y calidad de vida en general.

Las TIC como soluciones tecnológicas en el campo educativo permiten la interacción con actividades didácticas que integran lo visual, novedoso e interactivo; además incentivan el uso de cuadernos de trabajo online, facilitan la búsqueda, promueven las nuevas formas de enseñanza y la utilización de programas (software) para llevar lista de estudiantes, calificaciones, agenda de reuniones, etc. También facilitan el desarrollo de actividades prácticas del quehacer docente como elaborar crucigramas, sopa de letras, análisis de imágenes, talleres, diseño de evaluaciones, realización de juegos y videos, entre otros.

Sáenz (2010, p. 196) afirma que el propio aprovechamiento y buen uso de las tecnologías de información y comunicación en el medio escolar depende de la calidad y la idoneidad docente para asumir los nuevos desafíos pedagógicos que plantean su introducción en la escuela y el aula. Aprovechamiento, calidad e idoneidad que va enlazado con el uso, las prácticas pedagógicas y la importancia del significado que tiene el docente como pilar, mediador e innovador en la adquisición de capacidades desde una realidad objetiva y la experiencia subjetiva del estudiante en la apropiación de una realidad.

La inclusión de las TIC en el aula implica una práctica pedagógica reflexiva, investigadora y transformadora, en oposición a la práctica guiada por concepciones y creencias producto de la tradición cultural que permanecen implícitas.

Dentro de esta cultura debe emerger un profesorado autónomo, que piensa la educación a través de la reflexión sobre lo que hace en las aulas de clase; que toma decisiones con base en su interpretación de la realidad y crea situaciones nuevas a partir de los problemas de la práctica cotidiana con la finalidad de mejorarla o transformarla.

Diversas investigaciones demuestran que a pesar de los esfuerzos realizados en Colombia, aún persisten dificultades relacionadas con el conocimiento y la apropiación por parte de los docentes para emplear las TIC en sus prácticas pedagógicas. Un estudio realizado por la Universidad de los Andes muestra que 
en Colombia existen cerca de 262000 docentes que si bien puede que tengan acceso a un computador en el aula de clase, no le están sacando el mayor provecho pedagógico al mismo. Se ha comprobado además que, algunos docentes hacen un mayor uso de las TIC en actividades personales e instrumentales que se alejan de su sentido pedagógico.

Por consiguiente es necesario indagar sobre el uso que los docentes hacen de las tecnologías de la información y la comunicación en su práctica pedagógica desde diferentes niveles de apropiación: básico, medio y alto. Asimismo, sobre la familiarización, uso, integración, reorientación, y evolución indispensable para desarrollar nuevas forma de enseñanza aprendizaje en los docentes y estudiantes, y acercándolos a las dinámicas del mundo contemporáneo.

Desde esta perspectiva surge la interrogante: ¿Cómo están usando las TIC en sus prácticas pedagógicas los docentes rurales del departamento de Cundinamarca en Colombia?

\section{Contexto del estudio}

Con el objetivo de mejorar la calidad de la educación en Colombia el Ministerio de Educación Nacional4 (MEN) ${ }^{4}$ propone una política de calidad en la que se menciona la necesidad de atender, prioritariamente, a las prácticas pedagógicas de los docentes y el aprendizaje de los estudiantes.

4. De aquí en adelante, las autoras se referirán a esta institución a través de sus siglas.
A través del programa Nacional de innovación educativa con uso de TIC el Ministerio diseñó una propuesta de acercamiento al uso y apropiación de las mismas para docentes, con el objetivo de que ellos cuenten con una ruta para transitar, desde un nivel de apropiación personal hasta su apropiación pedagógica; reflejando un modelo de innovación educativa sostenible de uso y apropiación de las TIC.

En este marco, el Ministerio de Educación Nacional define en la Ruta de Apropiación de las TIC (2008, p.3), tres grandes ejes de política: a) Acceso a la tecnología; b) Acceso a los contenidos; y c) Uso y apropiación.

En esta ruta se plantean reflexiones sobre los diferentes horizontes de formación requeridos para la apropiación de TIC, trazándose como una estrategia para que los docentes construyan sus propios modos de acercamiento al desarrollo, de acuerdo con sus intereses y necesidades tanto personales como profesionales.

Se busca además que los docentes asistan a jornadas de capacitación que les planteen retos en relación con el conocimiento y el desarrollo de nuevas competencias en su quehacer pedagógico. Se pretende con estos procesos de formación lleven a los docentes colombianos a tener nuevas alternativas para integrar las TIC a la vida personal y profesional, de modo que puedan enfrentar los retos de la sociedad actual. 
Desde el 2007, el MEN de Colombia ha constituido acuerdos con aliados internacionales y nacionales para implementar la ruta, de tal manera que se apueste a fortalecer los procesos de formación de docentes en las TIC. Asimismo, que estos puedan hacer uso con sentido de las TIC, contribuyendo a cualificar los procesos personales, profesionales, institucionales y comunitarios.

El programa Computadores para Educar (CPE) del Gobierno Nacional, insertado en el Plan Vive Digital, surge bajo el liderazgo del Ministerio de Tecnologías de la Información y las Comunicaciones (MinTIC) como una estrategia que contribuye al cierre de la brecha digital y de conocimiento mediante el acceso, uso y aprovechamiento de las tecnologías de la información y la comunicación en las sedes educativas públicas del país. Todo esto a través de una propuesta de formación para profesores, que busca alcanzar transformaciones en los docentes, de tal manera que articule la formación y el acceso de las TIC mediante su apropiación pedagógica.

En la línea planeada por el Plan Nacional de Desarrollo, Computadores para Educar se une a la iniciativa en el tema de Crecimiento Sostenible y Competitividad especialmente a través de la estrategia "Innovación para la prosperidad" en la dimensión "Conocimiento e innovación", cuyos objetivos son apoyar la productividad y la competitividad del país; desarrollar contenidos y aplicaciones que ge- neran conocimientos y fortalecer las capacidades de los profesores de educación básica y media, especialmente en el sector rural.

Este programa, a través de la Estrategia de Formación y Acceso para la Apropiación Pedagógica de las TIC, dota a las sedes educativas del país, especialmente a aquellas ubicadas en las zonas rurales, con soluciones tecnológicas de escritorio o portátil; adicionalmente formando a los docentes con el fin de que accedan y se apropien pedagógicamente de las tecnologías.

En el marco de este proyecto es necesario partir del hecho que, la brecha para el acceso a las TIC inicia con la falta de alfabetización de uso de las mismas por parte de los maestros, sumado a las dificultades de conectividad y deficiencia del servicio eléctrico, indispensable para lograr el uso de herramientas informáticas orientadas a mediar los aprendizajes en los estudiantes.

El uso del ordenador y la introducción de las TIC, modifica las estructuras educativas, que en el contexto rural deben adaptarse a las formas de vida y de sociedad que allí se presentan. Así, el creciente acceso a la información -en cuanto a su rapidez y cantidad, a sus posibilidades de superar la distancia y el tiempo en la comunicación con otros- abre un espacio en la educación decisivo para el futuro. Investigar en él y adaptar poco a poco a la escuela y los procesos de enseñanza-aprendizaje es imprescindible si se tiene como propósito preparar a los estudiantes para la vida. 
En el contexto rural, esto tiene otras connotaciones y obliga a contemplar la relación intrínseca entre cultura y tecnología. No se puede desconocer que el medio rural en nuestra actual sociedad Colombiana, en muchas ocasiones, se encuentra olvidada. Existe escasez de medios, aislamiento, precariedad en la conformación de equipos docentes estables y preparados para el uso de las TIC; entre otras debilidades que mantienen a las poblaciones rurales en condición de vulnerabilidad y con desventajas de acceso al conocimiento.

\section{Práctica pedagógica}

De acuerdo con Zuluaga (2011), la práctica pedagógica es el escenario donde el maestro dispone todos aquellos elementos propios de su personalidad académica y personal. Desde lo académico, lo relacionado con su saber disciplinar y didáctico, como también el pedagógico a la hora de reflexionar sobre las fortalezas y debilidades de su quehacer en el aula. En lo personal, el maestro utiliza elementos como el discurso, relaciones intra e inter personal, ya que si no las tiene es seguro que no obtendrá éxito con la población a la cual va dirigido su conocimiento.

Actualmente, las prácticas pedagógicas se ven mediadas por las Tecnologías de la Información y Comunicación; dado que los gobiernos han optado por dotar las sedes educativas con computadores y tabletas. Sin embargo, tal y como se menciona en RINACE, "el computa- dor puede usarse según distintos modelos pedagógicos y las características de los mismos determinan sus efectos significativos o no, en el aprendizaje. Al mismo tiempo, resulta claramente el reconocimiento de que el computador puede usarse sin que haya innovación pedagógica” (2011, p 36). Es decir, el docente mantiene su acción pedagógica enmarcada en el modelo tradicional, e integra las TIC en algunos casos como fin y en otros como el medio visual para exponer la temática.

\section{Ambiente de aprendizaje}

De acuerdo con Roldán (2013), el ambiente de aprendizaje se concibe como el conjunto de condiciones o circunstancias físicas, sociales, económicas, biológicas, culturales, psicológicas, entre otras; que permiten y favorecen o dificultan y entorpecen el que los seres humanos, aprendan y enseñen. Es decir, que construyan e intercambien conocimientos y/o experiencias. Por lo tanto, interesa dar cuenta de las condiciones de las personas que se encuentran en ellos, del tipo y características de la interacción social que se establece; y de las especificidades del espacio que los acoge.

En cuanto al uso de las TIC en la práctica pedagógica, el papel del docente en relación con el ambiente de aprendizaje no es solo transmitir conocimientos si no pensar en cómo debe transformar su aula de clase en un ambiente dotado de soluciones tecnológicas -hardware y software-, permitiéndole desarrollar expe- 
riencias innovadoras en la enseñanza, para desarrollar habilidades de uso y aprovechamiento de las TIC en sus estudiantes.

De esta manera, los entornos de aprendizaje con soluciones tecnológicas que son usadas por los docentes y estudiantes con una intensión pedagógica, promueve un ambiente de aprendizaje donde los agentes que participan e interactúan asumen compromisos y autonomía en los procesos de aprehensión y generación de los conocimientos a través de la investigación. Ello permite el desarrollo del trabajo colaborativo, el aprovechamiento y utilización responsable de las TIC y de solución de problemas desde contextos reales. Por consiguiente, la tecnología amplía y enriquece el aprendizaje al contribuir al desarrollo de capacidades cognitivas de orden superior.

\section{La formación profesional de los docentes}

La integración de las tecnologías en el aula exige que los docentes desempeñen nuevos roles y transformen sus prácticas con nuevos planteamientos que les permitan responder con éxito a las exigencias de la sociedad del siglo XXI. Al mismo tiempo permite que sus estudiantes tengan la oportunidad de adquirir conocimientos significativos que les aporten a su crecimiento, desarrollo social y cultural.

Numerosas investigaciones muestran que es difícil transformar las prácticas pedagógicas de los docentes, y que aún se evidencian resistencias que conllevan a cambios superfi- ciales, que no reflejan procesos de reflexión e innovación en los procesos pedagógicos de los mismos.

En este sentido, es importante tener en cuenta que las transformaciones implican cambios culturales, cambios en el pensamiento y cambios en los modelos tradicionales.

Es claro que la sociedad actual está siendo bombardeada por nuevas formas de comunicación, hecho que se da en gran medida por el avance de las TIC. El uso de los celulares, computadores, entre otras herramientas tecnológicas están en expansión y son usados por niños y jóvenes.

Por consiguiente, uno de los desafíos más grandes en esta materia se centra en la formación del docente, puesto que es él quien desde las problemáticas reales y el contexto involucra las TIC para fortalecer los procesos de enseñanza-aprendizaje de sus estudiantes, rescatando el valor pedagógico, didáctico y epistemológico de ellas en la formación de los niños y jóvenes, para que sean agentes de transformación social.

En este estudio se plantea que la formación en las TIC debe pensarse como un proceso dinámico de ciclo continuo, que atienda a tres variables (saber que, saber ser, saber hacer); relacionando el conocimiento académico y práctico, convirtiéndolas en una forma para promover ese proceso de reflexión y cambios progresivos. Se menciona, además, como una 
manera de autoevaluación, una oportunidad para generar retos y sobre todo de transformar la manera en que el estudiante aprende, siendo este protagonista de su proceso, accediendo al conocimiento a través de estas herramientas.

\section{Uso de las TIC}

Marqués (2011) menciona que los principales usos de las TIC relacionadas con la educación son:

Alfabetización digital de los estudiantes (y profesores... y familias...). Uso personal (profesores, alumnos...); acceso a la información, comunicación, gestión y proceso de datos. Gestión del centro: secretaria, biblioteca, gestión de la tutoría de los alumnos. Uso didáctico para facilitar los procesos de enseñanza y aprendizaje. Comunicación con las familias (a través de la Web de centro...). Comunicación con el entorno. Relación entre profesores de diversos centros (a través de redes y comunidades virtuales) compartir recursos y experiencias, pasar informaciones, preguntas.

En los informes BECTA (2007), citados por Area (2008), los docentes usan las TIC fuera del aula para el desarrollo de tareas vinculadas con la planificación de su enseñanza, tales como:

- "La programación didáctica de la asignatura, de unidades didácticas o de lecciones mediante la utilización de procesadores de texto;
- la preparación de ejercicios o actividades que serán cumplimentadas por su alumnado;

- la elaboración de presentaciones multimedia;

- la navegación web para buscar información;

- la comunicación por email con otros colegas;

- la gestión administrativa: horarios, formularios burocráticos, boletines de calificaciones, etc." (Area, 2008 p 3).

Sin embargo, como lo afirma Area (2008) las actividades planificadoras como la "elaboración y producción de materiales didácticos digitales tales como webquest, edublogs, videoclips entre otros y el trabajo colaborativo con otros colegas para elaborar proyectos en las que las TIC se integran a las prácticas pedagógicas, son dejadas en un segundo plano" (p.3).

Asimismo, Area destaca que aun cuando se integran las TIC en las prácticas docentes, estas continúan respondiendo a modelos expositivos y/o tradicionales. (2008, p 4). Por ello, el uso de las TIC en el aula se orienta a:

- "Apoyar las exposiciones magistrales del profesor en el aula.

- Demandar al alumnado la realización de ejercicios o microactividades interactivas de bajo nivel de complejidad.

- Complementar o ampliar los contenidos del libros de texto solicitar al alumnado que busquen información en Internet. 
- Enseñar al alumnado competencias informáticas en el uso del software."(Area, 2008, p 5).

Por consiguiente, los niveles de integración y formas básicas de uso de la TIC del docente se describen como el aprovechamiento de estas tecnologías en su contexto educativo, a través de la implementación práctica en cada una de las situaciones que se generan en la comunidad educativa, tanto al interior de la escuela como en el entorno que la rodea. Esta es una forma de dar a conocer las experiencias del docente en una sociedad digital.

Desde esta perspectiva, es importante comprender que la llegada de una nueva tecnología a la escuela implica integrarla al currículo y al sistema escolar en general, mediante procedimientos pedagógicos operativos que aseguren su uso y utilidad en el proceso de enseñanza aprendizaje. Algunos autores consideran importante establecer la diferencia entre usar e integrar la tecnología. La primera hace referencia a emplearla para diversos fines, sin un propósito claro de apoyar un aprendizaje determinado. Por el contrario, en la segunda, se explica claramente una intención pedagógica, cómo aprender un concepto, un proceso, un contenido, en una disciplina curricular específica; teniendo en cuenta que el centro es el aprendizaje, en donde las TIC cumplen un papel de apoyo al mismo (Sánchez y Encalada, 2003).

Niveles de uso de las TIC Este aspecto es tratado por Torres (2011), quien plantea cuatro niveles de integración que vinculan el conocimiento y uso de las TIC por parte de los docentes: nivel de pre-integración, nivel de integración básica, nivel de integración medio y nivel de integración avanzada. 


\begin{tabular}{|c|c|c|c|c|c|c|c|c|c|c|c|c|c|c|c|c|c|}
\hline 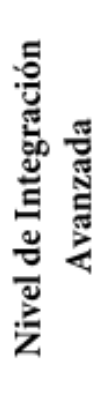 & 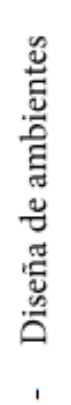 & 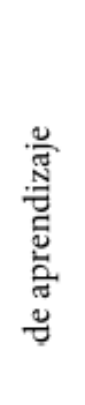 & 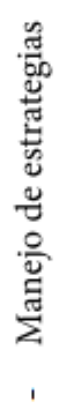 & 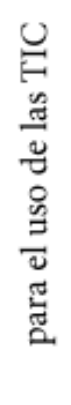 & 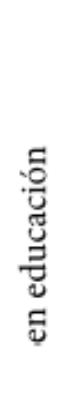 & 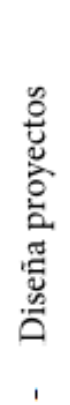 & 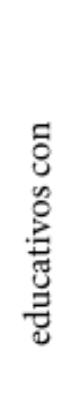 & 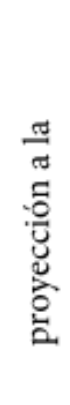 & 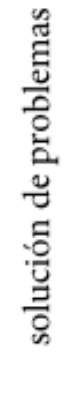 & & & & & & & & \\
\hline 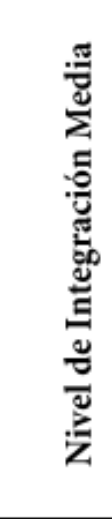 & 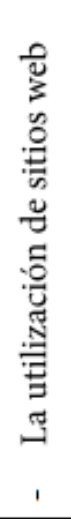 & 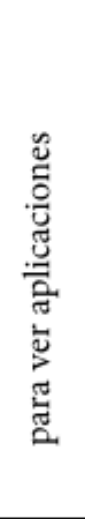 & $\begin{array}{l}\text { 总 } \\
\text { 总 } \\
\text { 总 }\end{array}$ & 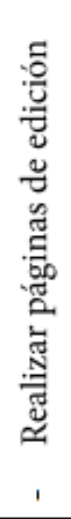 & 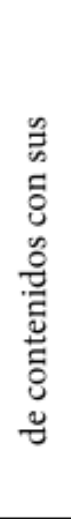 & 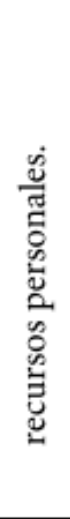 & 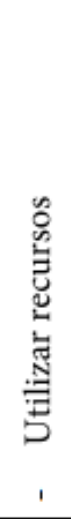 & 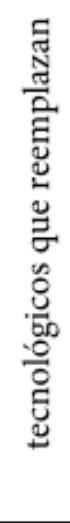 & 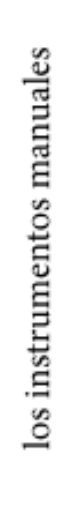 & 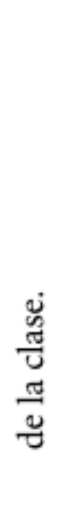 & 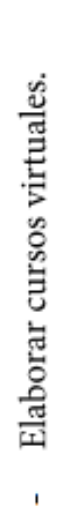 & 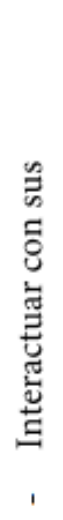 & 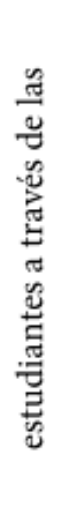 & 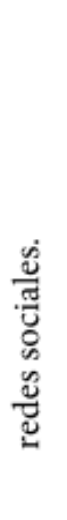 & 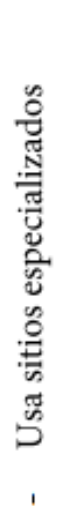 & 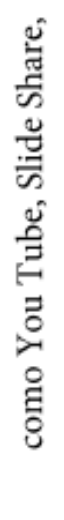 & 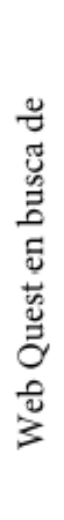 \\
\hline 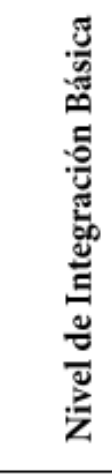 & 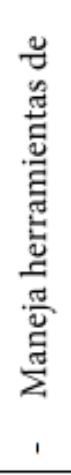 & 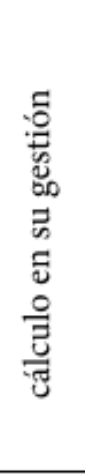 & $\begin{array}{l}\text { 总 } \\
\text { 窇 }\end{array}$ & 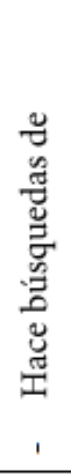 & 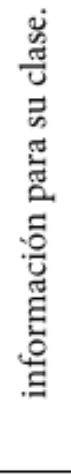 & 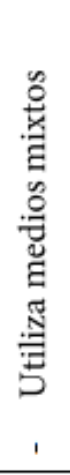 & 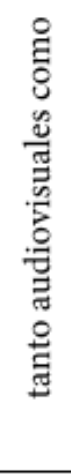 & 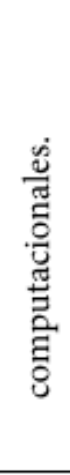 & 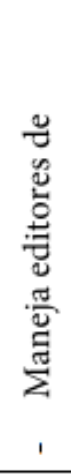 & 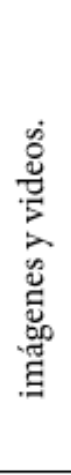 & 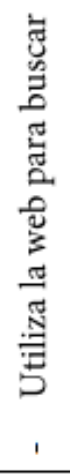 & 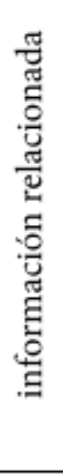 & 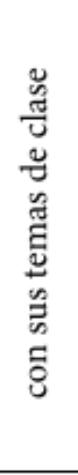 & & & & \\
\hline 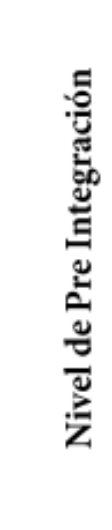 & 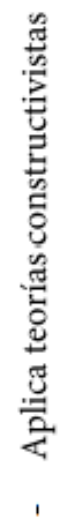 & 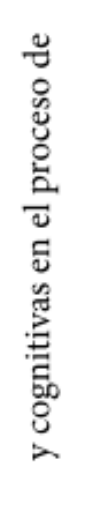 & 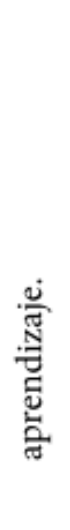 & 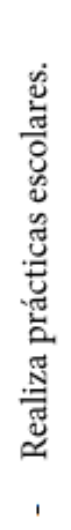 & 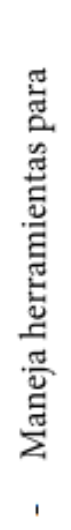 & 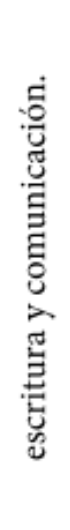 & 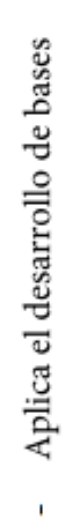 & 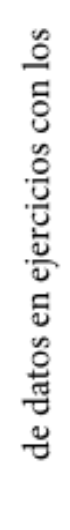 & 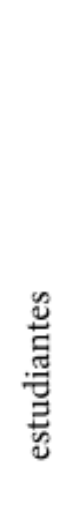 & & & & & & & & \\
\hline 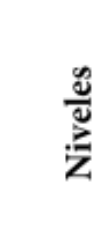 & & & & & & & & 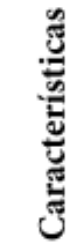 & 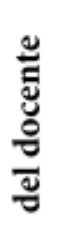 & & & & & & & & \\
\hline
\end{tabular}

María Patricia Gómez Becerra, Gloria Elizabeth Bernal De Felipe, Carolina Medrano León $\quad$ Volumen 2 


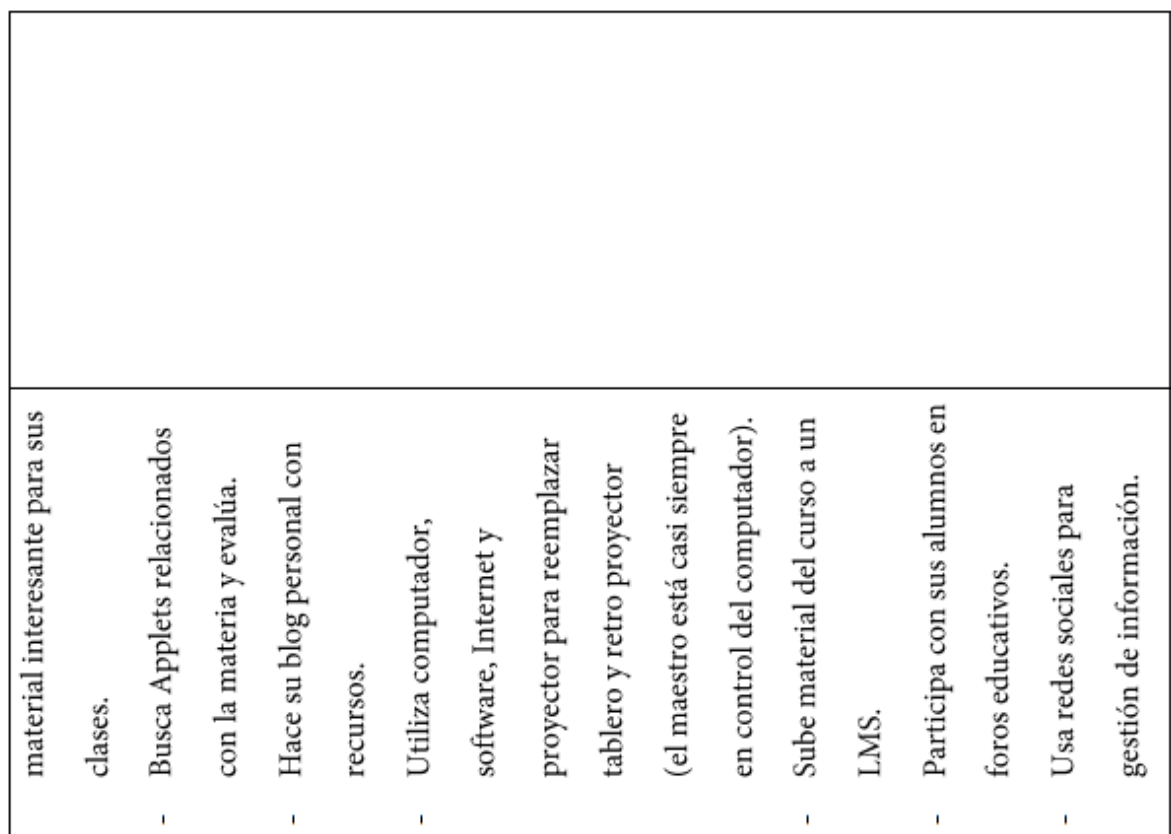

콩

U

ڤ્ટ

ن

$?$

ำ

$\pi$

5

晜

웅

过

.

$\stackrel{0}{0}$

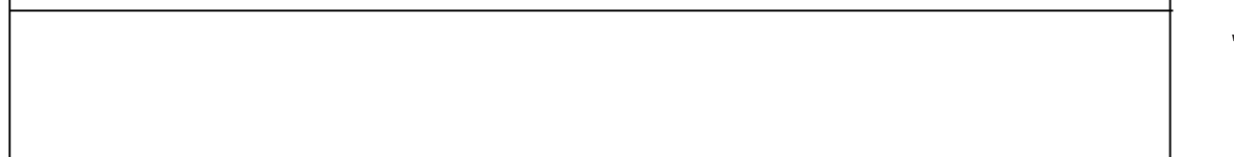

to

$\stackrel{\mathscr{Z}}{=}$

ปั

맘

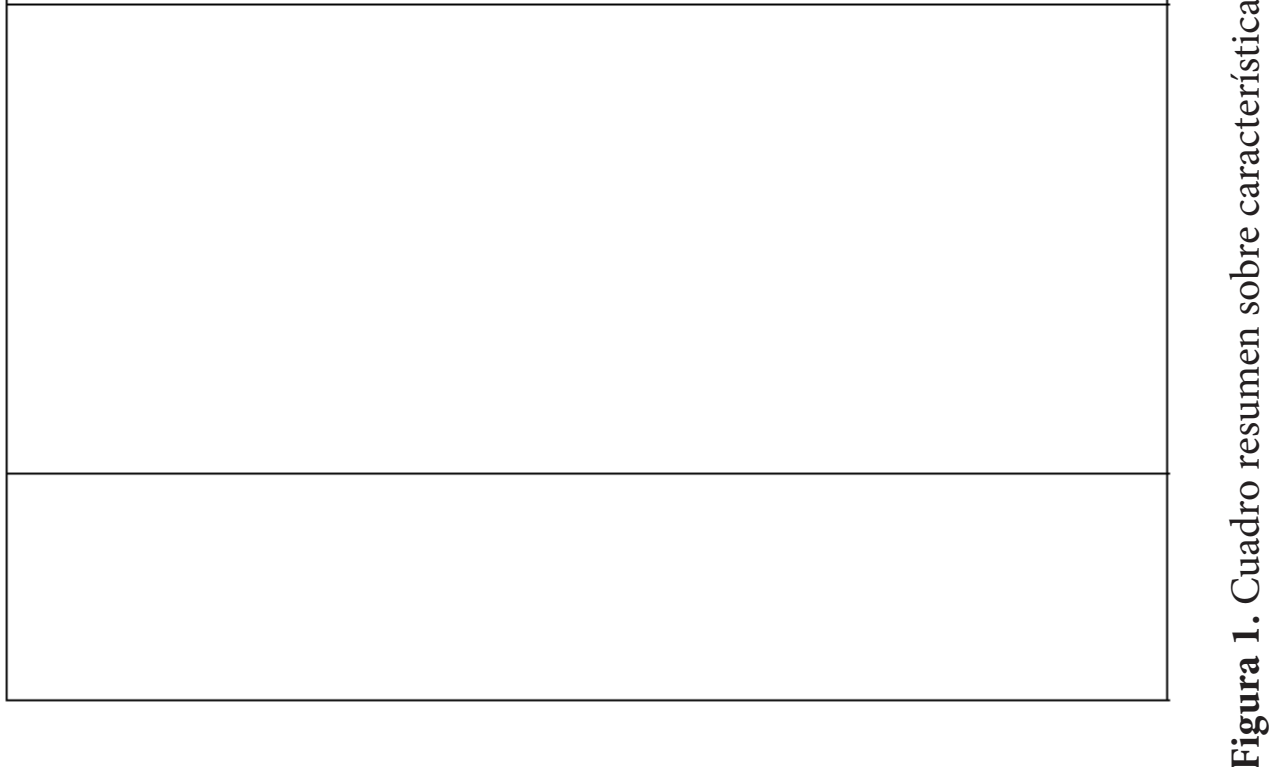


De acuerdo con lo anterior es necesario establecer en qué nivel de apropiación o acercamiento se encuentran los maestros en relación con el uso de las TIC, de manera tal que se puedan estructurar procesos de formación y acompañamiento que desde su realidad promuevan competencias y saberes que impacten su práctica pedagógica.

\section{Metodología}

Las instituciones educativas rurales en Colombia son aquellas que están ubicadas en entornos alejados de las ciudades o municipios principales de un departamento. Generalmente están ubicados en espacios geográficos de difícil acceso, con contextos que pueden propiciar altos índices de vulnerabilidad. De acuerdo con el Ministerio Nacional de Educación de Colombia, los problemas del sector rural son en gran parte causados por la baja cobertura (áreas rurales cobertura del 30\% comparada con 65\% de las áreas urbanas); la falta de calidad y pertinencia de un servicio educativo que no responde a las necesidades sociales. Todo ello sumado a otras dificultades entre las que sobresalen el aislamiento, el trabajo infantil, el bajo nivel de escolaridad de los padres, las tasas de deserción y repitencia, así como el número de niños que nunca ha sido atendido por el sector educativo. Por otro lado, se evidencia que la mayoría de las instituciones educativas rurales cuentan con un solo maestro que se encarga de atender varios cursos; y por consiguiente, asume el proceso de enseñanza de las diferentes áreas del conocimiento, lo cual puede constituirse como una fortaleza o una amenaza para el aprendizaje de los estudiantes; más aún cuando nos referimos al uso de las TIC en su práctica educativa.

\section{Diseño de instrumentos}

Desde una perspectiva metodológica mixta que incluye técnicas cualitativas y cuantitativas, los instrumentos se diseñaron a partir de la revisión teórica, observaciones en el aula, la construcción de categorías y ponderaciones, y una prueba piloto que se realizó con una muestra de docentes vinculados a instituciones educativas rurales.

A continuación se presenta como resultado y producto del estudio la siguiente tabla que especifica las categorías, indicadores y ponderación de los niveles de uso de las TIC en instituciones educativas rurales y que constituyó un insumo importante para el análisis de la información. 


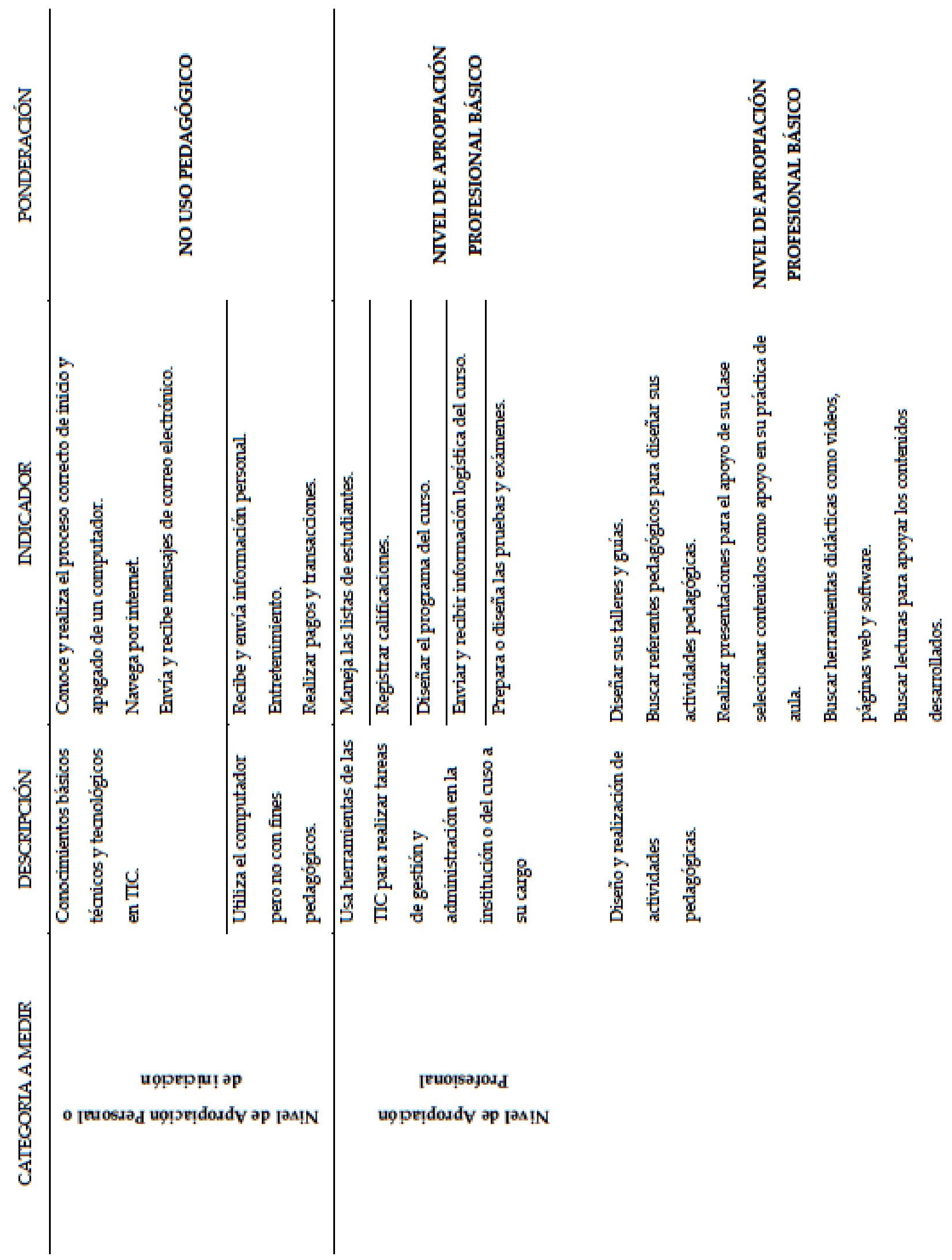

Volumen 2 Uso de las TIC en la Práctica Pedagógica de los Docentes Rurales en Colombia 

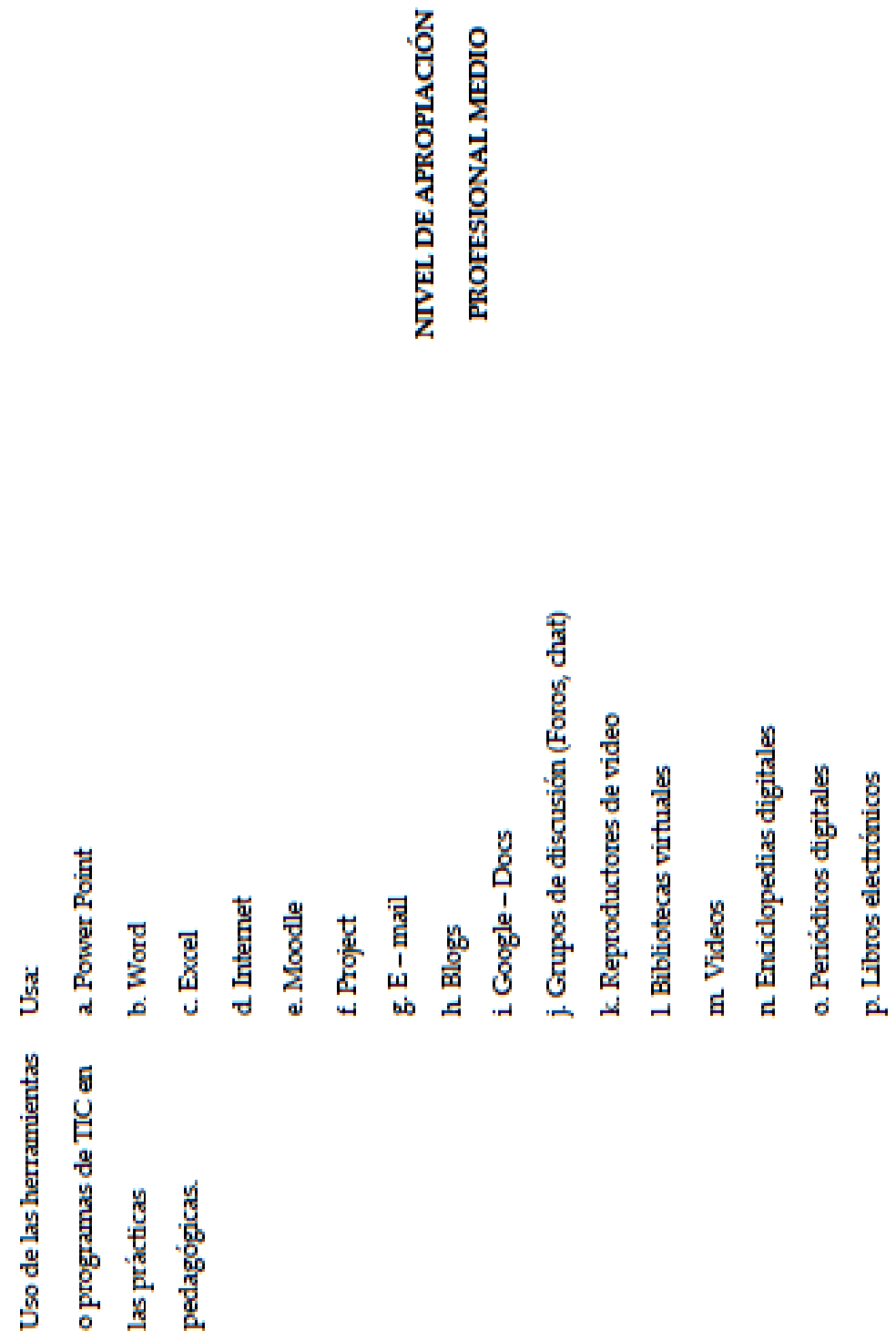

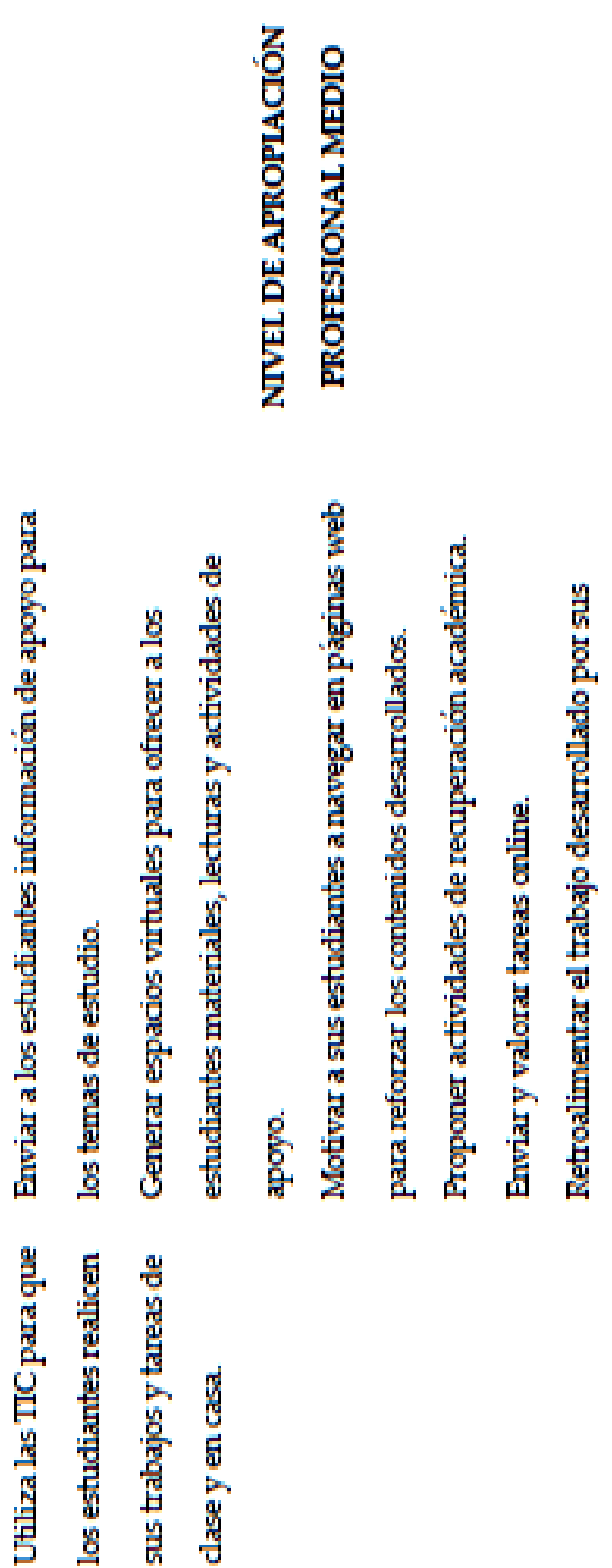
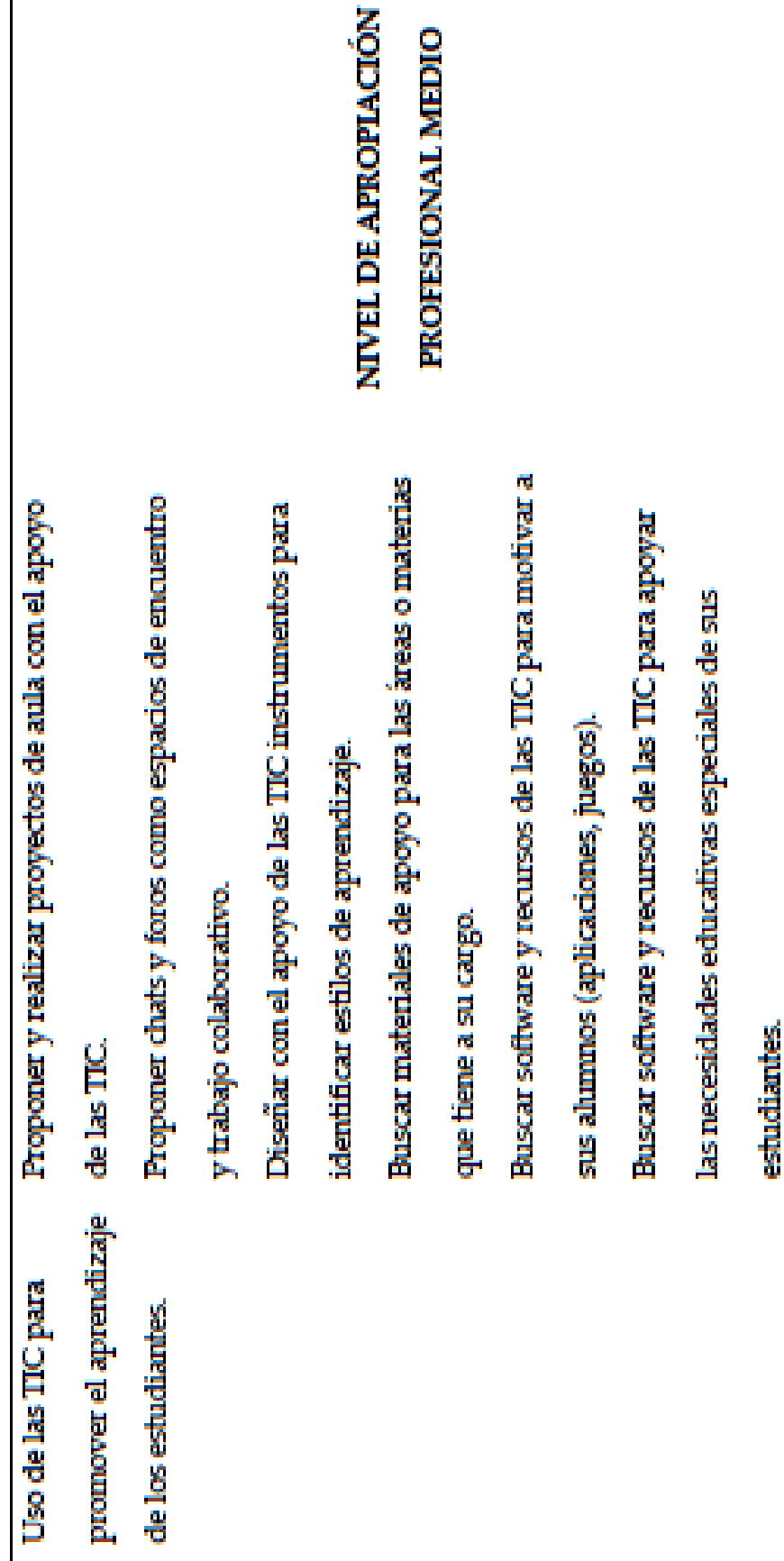


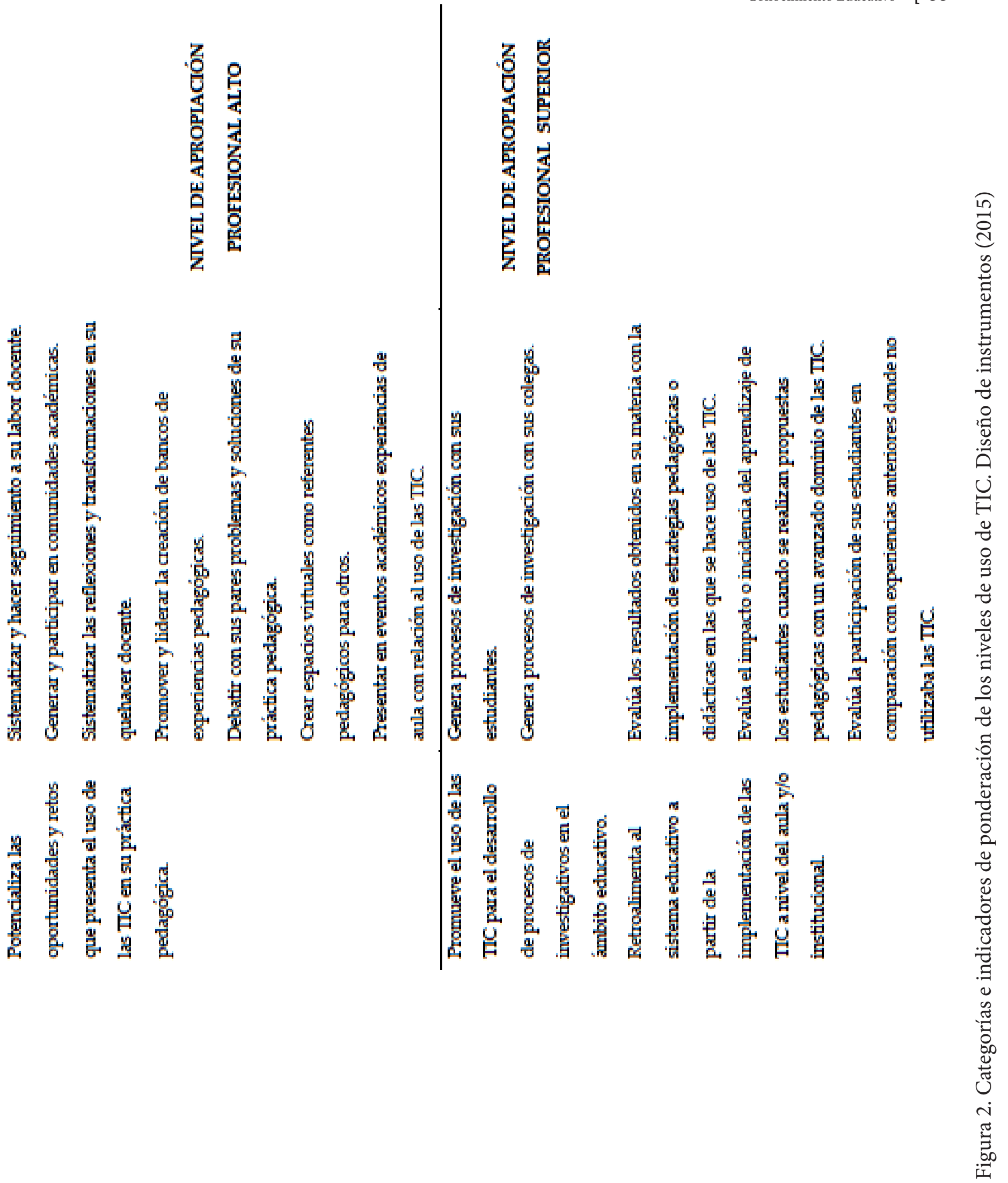

María Patricia Gómez Becerra, Gloria Elizabeth Bernal De Felipe, Carolina Medrano León $\quad$ Volumen 2 


\section{Resultados}

A continuación se presentan los resultados de acuerdo con los tres niveles de apropiación definidos para este estudio:

a. Categoría nivel de apropiación: personal o de iniciación

Tabla1. Resumen para nivel personal o de iniciación

\begin{tabular}{|c|c|c|c|c|c|c|}
\hline & \multicolumn{2}{|c|}{ Frecuentemente } & \multicolumn{2}{|c|}{ Ocasionalmente } & \multicolumn{2}{|c|}{ Nunca } \\
\hline & $F$ & $\%$ & $\mathbf{F}$ & $\%$ & $F$ & $\%$ \\
\hline Recibir y enviar correos electrónicos & 18 & 86 & 1 & 5 & 2 & 10 \\
\hline Navegar por internet & 14 & 67 & 5 & 24 & 2 & 10 \\
\hline Participar en redes sociales & 11 & 52 & 6 & 29 & 4 & 19 \\
\hline Entretenerse & 13 & 62 & 7 & 33 & 1 & 5 \\
\hline Realizar pagos y transacciones & - & - & 2 & 10 & 19 & 90 \\
\hline Realiza Compras & - & - & 3 & 14 & 18 & 86 \\
\hline Enterarse de noticias & 12 & 57 & 5 & 24 & 4 & 19 \\
\hline
\end{tabular}

Nota: Se muestra la frecuencia con que los docentes utilizan las TIC.

\section{b. Categoría nivel de apropiación: profesional medio}

Tabla 2. Resumen para nivel profesional medio

\begin{tabular}{|l|c|c|c|c|c|c|c|}
\hline & Frecuentemente & Ocasionalmente & \multicolumn{2}{c}{ Nunca } \\
\hline & F & $\%$ & F & $\%$ & F & $\%$ \\
\hline Power Point & 13 & 62 & 5 & 24 & 3 & 14 \\
\hline Word & 19 & 90 & 1 & 5 & 1 & 5 \\
\hline Excel & 12 & 57 & 6 & 29 & 3 & 14 \\
\hline Internet & 3 & 14 & 3 & 14 & 15 & 71 \\
\hline Moodle & - & - & 2 & 10 & 19 & 90 \\
\hline Project & - & - & 2 & 10 & 19 & 90 \\
\hline E-mail & 3 & 14 & 4 & 19 & 14 & 67 \\
\hline Blogs & 2 & 10 & 3 & 14 & 16 & 76 \\
\hline Google Docs & 2 & 10 & 2 & 10 & 17 & 81 \\
\hline Grupos de discusión & 1 & 5 & 3 & 14 & 17 & 81 \\
\hline Reproductores de video & 11 & 52 & 2 & 10 & 8 & 38 \\
\hline Bibliotecas virtuales & 2 & 10 & 4 & 19 & 15 & 71 \\
\hline Videos & 13 & 62 & 3 & 14 & 5 & 24 \\
\hline Enciclopedias digitales & 3 & 14 & 4 & 19 & 14 & 67 \\
\hline Periódicos digitales & 1 & 5 & 1 & 5 & 19 & 90 \\
\hline Libros Electrónicos & 2 & 10 & 1 & 5 & 18 & 86 \\
\hline Celular & 9 & 43 & 5 & 24 & 7 & 33 \\
\hline
\end{tabular}

Nota: Se confrontaron los datos medios con frecuencia de uso en la práctica pedagógica. 
Tabla 3. Resumen para promoción de tareas y trabajos mediante las TIC

\begin{tabular}{|c|c|c|c|c|c|c|}
\hline & \multicolumn{2}{|c|}{ Frecuentemente } & \multicolumn{2}{|c|}{ Ocasionalmente } & \multicolumn{2}{|c|}{ Nunca } \\
\hline & $\mathbf{F}$ & $\%$ & $\mathbf{F}$ & $\%$ & $\mathbf{F}$ & $\%$ \\
\hline $\begin{array}{l}\text { Enviar a los estudiantes información de apoyo } \\
\text { para los temas de estudio }\end{array}$ & - & - & 3 & 14 & 18 & 86 \\
\hline $\begin{array}{l}\text { Generar espacios virtuales para ofrecer a los } \\
\text { estudiantes materiales, lecturas y actividades } \\
\text { de apoyo }\end{array}$ & 3 & 14 & 1 & 5 & 17 & 81 \\
\hline $\begin{array}{l}\text { Motivar a sus estudiantes a navegar en páginas } \\
\text { web para reforzar los contenidos en clase }\end{array}$ & 4 & 19 & 1 & 5 & 16 & 76 \\
\hline $\begin{array}{llll}\begin{array}{l}\text { Proponer } \\
\text { académica }\end{array} & \text { actividades de recuperación } \\
\end{array}$ & 1 & 5 & 1 & 5 & 19 & 90 \\
\hline Enviar y valorar tareas online & - & - & 2 & 10 & 19 & 90 \\
\hline $\begin{array}{l}\text { Retroalimentar el trabajo desarrollado por sus } \\
\text { estudiantes }\end{array}$ & 4 & 19 & 2 & 10 & 15 & 71 \\
\hline
\end{tabular}

Nota: Se consultó a los docentes sobre la frecuencia con la que promueven trabajos y tareas entre los estudiantes.

Tabla 4. Resumen sobre indicador de utilización para aprendizaje

\begin{tabular}{|c|c|c|c|c|c|c|}
\hline & \multicolumn{2}{|c|}{ Frecuentemente } & \multicolumn{2}{|c|}{ Ocasionalmente } & \multicolumn{2}{|c|}{ Nunca } \\
\hline & $\mathbf{F}$ & $\%$ & $\mathbf{F}$ & $\%$ & $\mathbf{F}$ & $\%$ \\
\hline $\begin{array}{l}\text { Proponer y realizar proyectos de aula con el } \\
\text { apoyo de las TIC }\end{array}$ & 15 & 71 & 2 & 10 & 4 & 19 \\
\hline $\begin{array}{l}\text { Proponer chats y foros como espacios de } \\
\text { encuentro y trabajo colaborativo }\end{array}$ & 2 & 10 & 3 & 14 & 16 & 76 \\
\hline $\begin{array}{l}\text { Diseñar con el apoyo de las } \Pi \mathrm{IC} \text { instrumentos } \\
\text { para identificar estilos de aprendizaje }\end{array}$ & 6 & 29 & 5 & 24 & 10 & 48 \\
\hline $\begin{array}{l}\text { Buscar materiales de apoyo para las áreas o } \\
\text { materias que tiene a su cargo }\end{array}$ & 13 & 62 & 5 & 24 & 3 & 14 \\
\hline $\begin{array}{l}\text { Buscar software y recursos de las Tic para } \\
\text { motivar a sus alumnos (aplicaciones, juegos }\end{array}$ & 13 & 62 & 6 & 29 & 2 & 10 \\
\hline $\begin{array}{l}\text { Buscar software y recursos de las TIC para } \\
\text { apoyar las necesidades educativas de sus } \\
\text { estudiantes }\end{array}$ & 11 & 52 & 7 & 32 & 3 & 14 \\
\hline
\end{tabular}

Nota: Se confrontó la frecuencia del uso de las TIC en el aprendizaje de los estudiantes. 
Tabla 5. Resumen para indicador de desempeño

\begin{tabular}{|c|c|c|c|c|c|c|}
\hline & \multicolumn{2}{|c|}{ Frecuentemente } & \multicolumn{2}{|c|}{ Ocasionalmente } & \multicolumn{2}{|c|}{ Nunca } \\
\hline & $\mathbf{F}$ & $\%$ & $\mathbf{F}$ & $\%$ & $\mathbf{F}$ & $\%$ \\
\hline $\begin{array}{l}\text { Generar espacios virtuales que promueven } \\
\text { procesos de autoevaluación }\end{array}$ & - & - & 5 & 24 & 16 & 76 \\
\hline Diseñar instrumentos & 5 & 24 & - & - & 16 & 76 \\
\hline $\begin{array}{l}\text { Proponer protocolos y manuales de } \\
\text { procedimiento, normas o simulaciones }\end{array}$ & 1 & 5 & 1 & 5 & 19 & 90 \\
\hline
\end{tabular}

Nota: Frecuencia de uso de las TIC y su relación en el desempeño de los estudiantes.

\section{c. Categoría nivel de apropiación: profesional alto}

Tabla 6. Resumen para nivel profesional alto

\begin{tabular}{|c|c|c|c|c|c|c|}
\hline & \multicolumn{2}{|c|}{ Frecuentemente } & \multicolumn{2}{|c|}{ Ocasionalmente } & \multicolumn{2}{|c|}{ Nunca } \\
\hline & $\mathbf{F}$ & $\%$ & $\mathbf{F}$ & $\%$ & $\mathbf{F}$ & $\%$ \\
\hline $\begin{array}{l}\text { Sistematizar y hacer seguimiento a su labor } \\
\text { docente }\end{array}$ & 8 & 38 & 4 & 19 & 9 & 43 \\
\hline $\begin{array}{l}\text { Generar y participar en comunidades } \\
\text { académicas }\end{array}$ & 3 & 14 & 7 & 33 & 11 & 52 \\
\hline $\begin{array}{l}\text { Sistematizar las reflexiones y transformaciones } \\
\text { en su quehacer docente }\end{array}$ & 3 & 14 & 5 & 24 & 13 & 62 \\
\hline $\begin{array}{l}\text { Promover y liderar creación de bancos de } \\
\text { experiencias pedagógicas }\end{array}$ & 2 & 10 & 4 & 19 & 15 & 71 \\
\hline $\begin{array}{l}\text { Debatir con sus pares problemas y soluciones } \\
\text { de su práctica pedagógica }\end{array}$ & 4 & 19 & 4 & 19 & 13 & 62 \\
\hline $\begin{array}{l}\text { Crear espacios virtuales como referente } \\
\text { pedagógico }\end{array}$ & 2 & 10 & 4 & 19 & 15 & 71 \\
\hline $\begin{array}{l}\text { Presentar eventos académicos experiencias de } \\
\text { aula con relación al uso de las TIC }\end{array}$ & 3 & 14 & 3 & 14 & 15 & 71 \\
\hline
\end{tabular}

Nota: Frecuencia de uso de las TIC en relación a las oportunidades y retos de la práctica pedagógica. 


\section{d. Categoría nivel de apropiación: profesional superior}

Tabla 7. Resumen para nivel avanzado

\begin{tabular}{|c|c|c|c|c|c|c|}
\hline & \multicolumn{2}{|c|}{ Frecuentemente } & \multicolumn{2}{|c|}{ Ocasionalmente } & \multicolumn{2}{|c|}{ Nunca } \\
\hline & $F$ & $\%$ & $F$ & $\%$ & $\mathbf{F}$ & $\%$ \\
\hline $\begin{array}{l}\text { Generar procesos de investigación con sus } \\
\text { estudiantes sobre la utilización de las TIC }\end{array}$ & 3 & 14 & 9 & 43 & 9 & 43 \\
\hline $\begin{array}{l}\text { Generar procesos de investigación con sus } \\
\text { colegas sobre las TIC en las prácticas } \\
\text { pedagógicas }\end{array}$ & 2 & 10 & 6 & 29 & 13 & 62 \\
\hline $\begin{array}{l}\text { Elaborar y publicar con el apoyo de las TIC } \\
\text { investigaciones y artículos sobre su práctica } \\
\text { pedagógica }\end{array}$ & 2 & 10 & 2 & 10 & 17 & 81 \\
\hline
\end{tabular}

Nota: Relación frecuencia-promoción de TIC en procesos investigativos en el ámbito educativo.

Tabla 8. Resumen para retroalimentación con base en la utilización de TIC

\begin{tabular}{|c|c|c|c|c|c|c|}
\hline $\begin{array}{l}\text { Evaluar el impacto o incidencia del aprendizaje } \\
\text { de los estudiantes cuando se realizan } \\
\text { propuestas pedagógicas con un avanzado } \\
\text { dominio de TIC }\end{array}$ & 2 & 10 & 5 & 24 & 14 & 68 \\
\hline $\begin{array}{l}\text { Evaluar el desempeño de sus estudiantes en } \\
\text { comparación con experiencias anteriores donde } \\
\text { no utilizaba las TIC }\end{array}$ & 5 & 24 & 5 & 24 & 11 & 52 \\
\hline $\begin{array}{l}\text { Proponer alternativas de mejoramiento de la } \\
\text { calidad educativa a partir de uso del TIC }\end{array}$ & 5 & 24 & 5 & 24 & 11 & 52 \\
\hline $\begin{array}{l}\text { Generar conocimientos relevantes en } \\
\text { innovadores que aporten a la transformación } \\
\text { de los procesos pedagógicos, vinculando a } \\
\text { estudiantes, padres de familia pares e } \\
\text { institución general }\end{array}$ & 3 & 14 & 5 & 24 & 13 & 62 \\
\hline
\end{tabular}

Nota: Relación frecuencia de uso - retroalimentación por parte de los docentes. 


\section{Discusión}

El nivel de formación y experiencia de los docentes no sería un factor decisivo en los procesos de uso de las TIC, dado que un 95\% de los docentes de la muestra son licenciados; es decir, profesionales de la educación, lo que supone un desarrollo de competencias en la implementación de estas herramientas en su vida profesional, académica y cotidiana. Tampoco se relaciona con la edad de los docentes, puesto que la mayoría se encuentra entre los 20 y 45 años.

Un alto porcentaje de los docentes rurales participantes en el estudio se encuentran en un nivel de apropiación básico en el uso de las TIC, dado que en su práctica predominan actividades como: recibir y enviar correos electrónicos, manejar listas de estudiantes, registrar calificaciones, diseñar programas académicos, preparar o diseñar pruebas y exámenes, diseñar talleres y guías, buscar referentes pedagógicos para diseñar sus actividades pedagógicas; realizar presentaciones para el apoyo de sus clases, buscar herramientas didácticas como videos, páginas web y software, así como buscar lecturas para apoyar los contenidos desarrollados.

Con respecto al uso de apropiación profesional medio es importante resaltar que se evidencia un avance y transición de algunos de los docentes a este nivel, principalmente en el uso que hacen de las TIC para proponer y realizar proyectos de aula, motivar a sus estudiantes a navegar en páginas web para reforzar los contenidos de la clase, buscar software y recursos de las TIC para motivar a sus alumnos y apoyar las necesidades educativas de sus estudiantes.

Los resultados dan cuenta que los docentes se están movilizando de un nivel a otro frente al llamado de hacer un uso profesional alto de las TIC, principalmente en la formulación, seguimiento y desarrollo de proyectos educativos. Sin embargo, es claro que esta movilización, requiere de una infraestructura en las instituciones educativas de las áreas rurales, que garanticen el acceso a las herramientas y medios tecnológicos, para que los docentes hagan un uso efectivo de las TIC en sus prácticas pedagógicas.

Cabe destacar que aunque con bajos porcentajes, algunos de los docentes han empezado a involucrar prácticas que hacen parte del nivel de uso profesional superior, tales como la generación de procesos de investigación con sus estudiantes sobre procesos de utilización de las TIC; evaluación de resultados obtenidos con la implementación de estrategias pedagógicas en las que se hace uso de ellas, y la evaluación del desempeño de sus estudiantes en comparación con experiencias anteriores donde no utilizaban las TIC.

En las escuelas rurales estudiadas predomina un tipo de enseñanza tradicional, en la que el 
uso de los libros de texto, circulares y cuadernos sigue siendo una constante.

Posiblemente una de las causas del bajo nivel de uso de las TIC se relaciona con la falta de recursos y acceso a internet, dada la ubicación de las escuelas en zonas rurales, donde la conectividad y acceso es limitado.

Los resultados obtenidos en el estudio generan un interrogante frente a la participación de los directivos docentes en el reto de propiciar las condiciones institucionales necesarias para promover es sus comunidades educativas una cultura digital.

Se evidenció que la adquisición de conocimientos y habilidades en el uso de las TIC, en el ámbito pedagógico, se centra fundamentalmente en los espacios académicos que tienen como propósito específico enseñar sobre su uso. Pero, ni siquiera estos espacios académicos son considerados en algunos de los currículos de las escuelas rurales.

De acuerdo con lo anterior, sería importante indagar sobre las posibilidades que se generan a partir de la incorporación de esta área del conocimiento en el currículo de las instituciones educativas rurales desde los niveles preescolar, y no solo desde la educación básica primaria.

Queda pendiente profundizar en las actitudes, representaciones y concepciones que los docentes tienen del uso de las TIC en los pro- cesos pedagógicos. Es decir, qué utilidad ven en ellas, su deseo de incorporarlas y considerarlas como herramientas que proporcionan diferentes oportunidades de aprendizaje y enseñanza en el aula. Pero al parecer, los procesos de formación en el uso de estas solo han permeado el discurso de los maestros sin trascender a su quehacer pedagógico y cotidianidad.

Finalmente, sería importante generar rutas o líneas de acción que propicien el seguimiento y acompañamiento a los docentes beneficiados por estrategias de capacitación en el uso de las TIC; más cuando estas son lideradas desde la política pública y las entidades gubernamentales como es el caso de la Estrategia de Formación y Acceso para la Apropiación Pedagógica de las TIC en Colombia. Este acompañamiento en su quehacer pedagógico afianzaría una transición hacia un nivel de apropiación profesional de las TIC, proyectándolos como agentes educativos activos, capaces de promover el uso de las mismas, para el desarrollo de procesos investigativos en el ámbito educativo. Al mismo tiempo, ello los llevaría a asumir el reto de retroalimentar el sistema educativo para así avanzar en la búsqueda de una educación de calidad. 


\section{Referencias}

Almerich, G.; Suárez J.; Jornet, J. y Orellana, M. (2011).Las competencias y el uso de las Tecnologías de Información y Comunicación (TIC) por el profesorado: estructura dimensional. Revista electrónica de investigación educativa. REDIE vol.13 no.1 Ensenada 2011. Recuperado de: http://www.scielo.org.mx/scielo.php?pid=S1607-40412011000100002\&script=sci_arttext

Area M., Manuel. (2008). Innovación pedagógica con TIC y el desarrollo de las competencias informacionales y digitales. Artículo publicado en la Revista Investigación en la escuela, no 64, 2008, Facultad de Educación. Universidad de La Laguna. págs. 518. Recuperado de: http://manarea.webs.ull.es/articulos/art16_investigacionescuela.pdf

BID (2011). Modelos Uno a Uno en América Latina y el Caribe. Panorama y perspectivas. Recuperado de: http://idbdocs.iadb.org/wsdocs/getdocument.aspx?docnum=35838865.

Claro, M. (2010).La incorporación de tecnologías digitales en educación. Modelos de identificación de buenas prácticas. Chile: Naciones Unidas.

Coll, C. y Monereo, C. (2008). Psicología de la educación virtual. Aprender y enseñar con las tecnologías de la información y la comunicación. Madrid: Morata.

Díaz, B. F. (2010) Integración de las TIC en el currículo y la enseñanza para promover la calidad educativa y la innovación. Pensamiento Iberoamericano. 7 (2), pp.129-149.

Dorfsman, M. (2012) La profesión docente en contextos de cambio: el docente global en la sociedad de la información. RED-DUSC. Revista de Educación a Distancia-Docencia Universitaria en la Sociedad del Conocimiento. Número 6. Murcia: Universidad de Murcia. Recuperado de http://www.um.es/ead/reddusc/6/marcelo_dusc6.pdf

Fandos, G. M.; Jiménez, J, M y González, Á, P. Estrategias didácticas en el uso de las Tecnologías de la Información y la Comunicación. Revista Acción Pedagógica A, Vol., 11. No. 1., p. 28-39.

Ferro, S. C; Martínez, A, I; Otero N, M, C. (2009) Ventajas del uso de las TIC en el proceso de enseñanza-aprendizaje desde la óptica de los docentes universitarios españoles. Facultad de Ciencias Económicas y Empresariales Universidad de Vigo. Edutec. Revista Electrónica de Tecnología Educativa. No. 29. 
González, A, F., Carretero, G, M., Escudero, V, J., y Arranz, G, O. (2011). Niños 2.O, una experiencia formativa en actitudes y valores para el profesorado ante la Web 2.0 y TIC. TeoríA De La EducacióN: EducacióN Y Cultura En La Sociedad De La Información, 12(4), 25-38. Recuperado de http://revistas.usal.es/ revistas_trabajo/index.php/revistatesi/article/view/8524

Guerra, M y Jordán, V. (2010). Políticas públicas de Sociedad de la Información en América Latina: ¿una misma visión? Recuperado de http://www.cepal.org/SocInfo

ISTE (2009). Estándares Nacionales (EEUU) de Tecnologías de Información y Comunicación (TIC) para directivas escolares. Ed. ISTE. Traducido por Eduteka. Recuperado de http://www. eduteka.org/pdfdir/EstandaresDirectivosNETS2009.pdf

Jaramillo, P; Castañeda, P; Pimienta, M. (2009) Qué hacer con la tecnología en el aula: inventario de usos de las TIC para aprender y enseñar Educación y Educadores. Vol. 12, Núm. 2 pp. 159-179 Universidad de La Sabana. Colombia.

Ministerio de Educación. (2010). Las TIC en la Educación Obligatoria: de la política y la práctica. Revista de educación No, 352 Madrid España. Recuperado de: http://books.google.es/bo oks?hl=es\&lr=\&id=dZ47AezyxawC\&oi=fnd\&pg=PA77\&dq=el+uso+de+las+tic+en+el+aula \&ots=R6zALW9W5Z\&sig=lRr48FT-Li6LvvOkSC4PlJWUM1U\#v=onepage\&q=el\%20uso\%20 de\%20las\%20tic\%20en\%20el\%20aula\&f=false

Porlán, R., Martin Del Pozo, R., Rivero, A., Harres, J., Azcarate, P. y Pizzato, M. (2010). El cambio del profesorado de ciencias I: marco teórico formativo. Enseñanza de las Ciencias, 28 (1), p. $31-46$

RINACE. (2011) Las tecnologías de la información y la comunicación en la escuela. Revista Iberoamericana de Evaluación Educativa, Vol. 4, Número 2 Recuperado de: http://rinace.net/ riee/numeros/vol4-num2/Riee\%204,2.pdf

Rodríguez, Z, E. (2010). El Plan Ceibal en las Escuelas Públicas del Uruguay. Revista Novedades Educativas, 236, pp.53-59, Medio de divulgación: Buenos Aires, Argentina. Recuperado de http://www.uruguayeduca.edu.uy

Roldán, O. (2013). Seminario Ambientes Educativos. Maestría en Educación y Desarrollo Humano. Convenio Universidad de Manizales - Cinde. 
Ruiz, O, J.I. (2012). Metodología de la Investigación Cualitativa. Universidad de Deusto.

Sánchez, S. (2012). Tecnologías de la información y la Comunicación en la Formación de políticas actuales, Revista Educación y Desarrollo Social Vol. 6 No 2.

Tejedor, F. (2010). Aportaciones de las TIC al desarrollo social. En Boza, A., Méndez, J., Monescillo, M. y De la O Toscano, M. Educación, Investigación y Desarrollo Social. Madrid: Narcea. UNESCO (2008). Estándares UNESCO de competencia en TIC para docentes.

UNESCO (2011). Estándares UNESCO de competencia en TIC para docentes.

Vaillant, D. (2013) Integración de TIC en los sistemas de Formación Inicial y continua para la Educación Básica en América Latina, UNICEF

Vázquez, M, A. (2013) Relación entre el enfoque de aprendizaje en el rendimiento académico universitario. Un estudio de caso. Revista de Medios y Educación. No 42 Universidad de Sevilla. Escuela Técnica Superior de Ingeniería de Edificación. Departamento Construcciones Arquitectónicas-II. Recuperado de http://acdc.sav.us.es/pixelbit/images/stories/p42/42. pdf $\#$ page $=115$ 